\title{
PLC Based PID for Wrapping Machine Tension Control
}

\author{
Sujono $^{1}$, Muhtadin $^{2}$ \\ \{ sujono@budiluhur.ac.id ${ }^{1}$, mochtadind@gmail.com² ${ }^{2}$, \\ Electrical Engineering Universitas Budi Luhur, Jakarta, Indonesia ${ }^{1,2}$
}

\begin{abstract}
The process of wrapping with plastics to better ensure the safety of the goods are mostly done, such as goods before being put into the trunk of the plane or when the industrial product to be delivered in long distances. In the wrapping process, the tension of the tension of the plastic (wrapping material) is very important to keep its value so that it will produce a wrapping that is in accordance with the desired. In this paper will be presented about the implementation of PID (Proportional Integral Differential) controller for tension control on wrapping machine. The system consists of a three phase induction motor as a drive in the unwinder and rewinders, PIDs of the PLC, inductive proximity as a plastic tension sensor, and a PC for data entry and system monitoring. The tension control of the plastic wrapping material is carried out by manipulating the unwinder velocity for the rewinder speed which is maintained at a constant speed. The speed difference between the two motors (unwinder and rewinder) will produce tension. To get good control performance, PID parameter tuning is required. From tuning using 3 methods (reaction curve method, tuning PID TIA PORTAL tool and Dotx PID tuner application), the best result resulted with $3.3 \%$ overshoot response, 1.7 second rise time, $3.2 \%$ settling time second, there is 1 oscillation and steady state error of $0.263 \%$.
\end{abstract}

Keywords: Control system, PID, tension, PLC, wrapping.

\section{Introduction}

In the manufacturing industry, often the product must be delivered in great distances. To keep the product from possible damage during the delivery process, it is often done by wrapping using plastic materials. Similar process is also often we find at the airport, where passengers do the wrapping of bags or luggage before it is put into the trunk of the plane.

The processing and packing industries are growing nowadays. Various efforts are made by automating the processes that exist in the industry. Changes are made from conventional ways of being integrated and forward as a whole. The technology in packing production will be an important role in improving productivity. Findings in the field of packing automation will be very beneficial to the processing and packing industries [1].

One of the most important things in the process of thin and flexible materials, such as thin plastics for wrapping, is to obtain a result of flawless wrapping or damage to plastic as wrapping materials. Damage can occur as a result of the level of tension thin plastic material exceeds the elasticity limit, so it is important to control the level of tension thin plastic material [2].

Other research has also been done on the importance of wire tension setting on the motor coil winding process automatically.In general, the process of coil winding motor using the 
device in the form of passive arm and braking hysteresis to keep the tension of the wire material.This passive arm device is unable to react quickly as the speed of winding increases.Sliding mode control is done by using an interference observer to estimate the strain of the wire [3].

In the process of rolling thin and flexible materials, problems often arise. It is as found for example in the rolling process of paper and plastic. The problem is because the inertia and radius slowly during transportation will affect the value of the spring constant of the material [4]. It is required to control precisely to the value of tension continuously in dynamics system [5].

During wrapping process, many variables must be controlled, one of them is the tension level of the plastic as wrapping material. This is done so that the quality of the wrapping process is good, not torn, evenly and tightly. Tension controller is a part that serves to tighten and relax the pull of plastic against items to be wrapped [2].

In this study will be discussed about the simulation of tension control system with PID controller on wrapping machine. The system used in the simulation consists of two three phase induction motors that functioned as a wheel drive plastic sheet. Tension control is done by manipulating unwinder roller motor at a certain speed which will cause adjustment of the speed of stretching of plastic material, so that will get the level of tension as desired. Motor speed setting is done by manipulating the frequency of the motor supply voltage generated from the inverter.

\section{Mechanical System on Wrapping Machine}

In figure 1 shows the path of plastic starting from the unwinder to the items to be wrapped that serves as a rewinder. First from plastic roll to roll unwinder then roll support unwinder. Roll support unwinder is useful for pressing plastic to roll unwinder so that plastic tension (tension) can be as expected (more effective in controlling tension). Then from roll support unwinder to roll dancer tension that can move forward or backward according to tension (tension) of plastic. From the tension dancer roll then past the supporting roll and directly wrap the items that are already on the rewinder board.

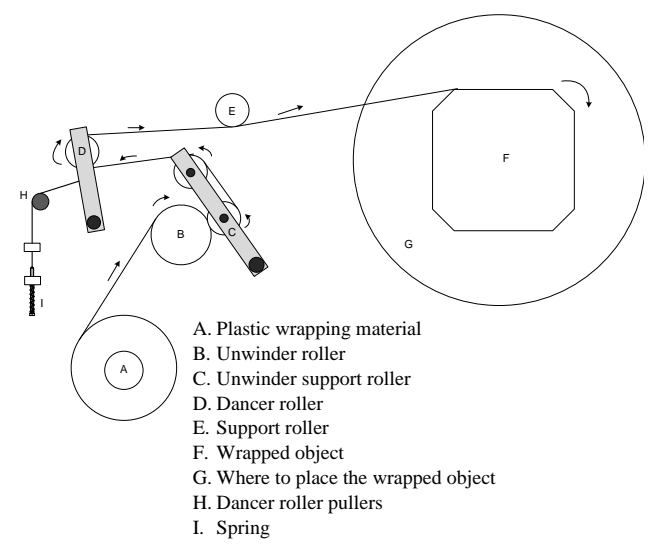

Fig. 1. Wrapping material path. 


\subsection{Tension on Plastic Sheet asWork piece}

The work piece in the form of plastic sheet has elastic properties. The elasticity of the plastic sheet is expressed in $\mathrm{K}$ and $\mathrm{D}$ constants [6]. As the work piece shifts along the work station, the plastic elasticity causes tension on the plastic sheet [7]. For more details can be seen in Figure 2.

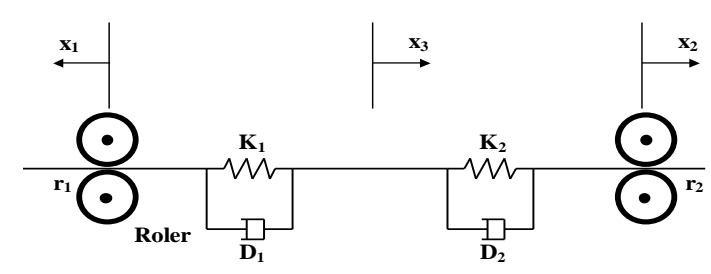

Fig. 2. Elasticity of work piece.

The equation of the tension level on the plastic sheet located between the roller 1 and the 2 nd roller is:

$$
\begin{aligned}
& f=K_{1}\left(x_{3}+x_{1}\right)+D_{1}\left(\frac{d x_{3}}{d t}+\frac{d x_{1}}{d t}\right) \\
& f=K_{2}\left(x_{2}-x_{3}\right)+D_{2}\left(\frac{d x_{2}}{d t}-\frac{d x_{3}}{d t}\right)
\end{aligned}
$$

$$
\begin{array}{ll}
\mathrm{f} & : \text { tension or tension (Newton) } \\
\mathrm{v} & \text { : rate of shift }(\mathrm{m} / \mathrm{sec}) \\
\mathrm{K}_{1}, \mathrm{~K}_{2} & \text { : flexibility constant }(\mathrm{N} / \mathrm{m}) \\
\mathrm{D}_{1}, \mathrm{D}_{2} & \text { : damping factor }(\mathrm{N} /(\mathrm{m} / \mathrm{sec}) \\
\mathrm{x}_{1}, \mathrm{x}_{2}, \mathrm{x}_{3} & \text { : magnitude of } \operatorname{shift}(\mathrm{m})
\end{array}
$$

The equation for the rate of plastic sheet shifts in the middle between roler 1 and roler 2 can be expressed as follows [8]:

$$
x_{3}=\frac{x_{2}-x_{1}}{2}
$$

Substituting equation (3) to equation (2) will then be obtained:

$$
f=\frac{K_{2}}{2}\left(x_{1}+x_{2}\right)+\frac{D_{2}}{2}\left(\frac{d x_{1}}{d t}+\frac{d x_{2}}{d t}\right)
$$

The relationship between the rotational speed of the roler and the rate of plastic sheet shift:

$$
\frac{d x_{1}}{d t}=r_{1} \cdot \omega_{1} \quad \text { dan } \quad \frac{d x_{2}}{d t}=r_{2} \cdot \omega_{2}
$$


For $\mathrm{K}_{1}=\mathrm{K}_{2}=\mathrm{K}$ and $\mathrm{D}_{1}=\mathrm{D}_{2}=\mathrm{D}$, if equation (5) is substituted to equation (4) it will be obtained:

$$
f=\frac{K}{2} \int r_{1} \cdot \omega_{1} d t+\frac{D}{2} r_{1} \cdot \omega_{1}+\frac{K}{2} \int r_{2} \cdot \omega_{2} d t+\frac{D}{2} r_{2} \cdot \omega_{2}
$$

\subsection{Tension Control System Block}

The design of the control system to be discussed in this paper is as presented in Figure 3 .

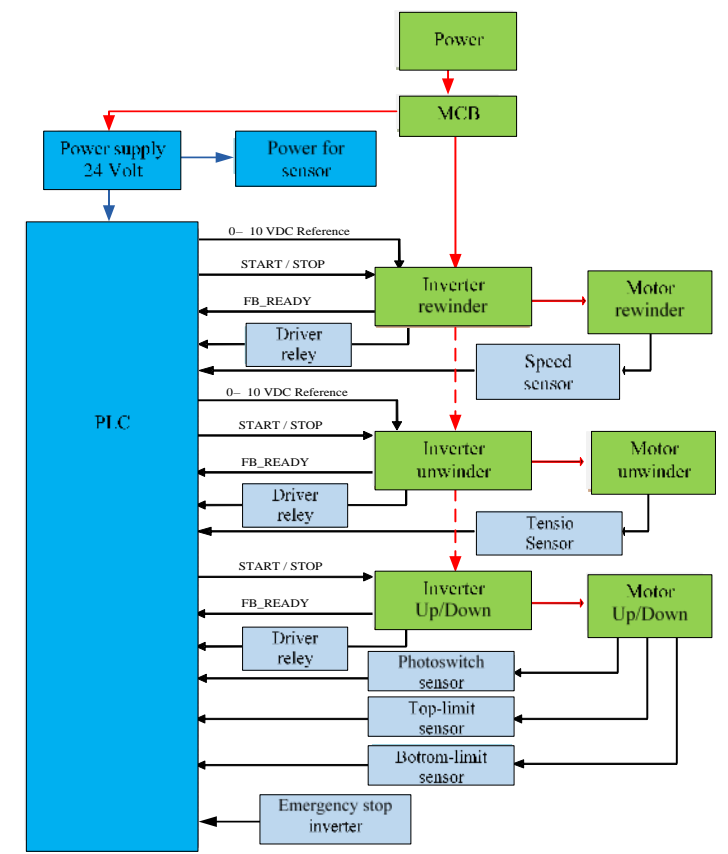

Fig. 3. Block Diagram of the System.

SIEMENS S7-1200 PLC is used as a regulator of the entire process that occurs in the system [9]. The three phase induction motors are used to drive the existing roler, by setting the speed through the working frequency of the motor working voltage that generated from the inverter. Proximity switch is used to detect the speed of motor rotation. Tension control algorithm uses PID with parallel structure.

\subsection{Tension Sensor}

Tension on the plastic material is detected by adding a free roller (dancer roller). Changes in the level of tension on the plastic material will result in changes in the compression force of the plastic sheeting material to the dancer roller. 


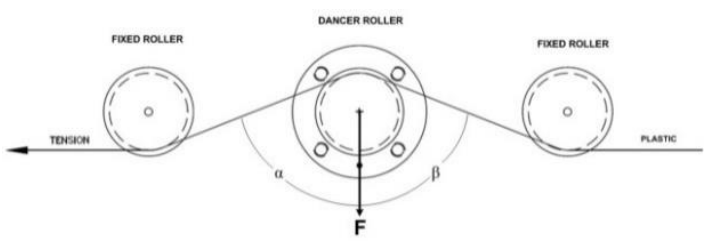

Fig. 4. Pressure force with the angle of the shaper.

$$
F=f \cdot \cos a+f \cdot \cos b
$$

$$
\begin{array}{ll}
\mathrm{F} & =\text { press force }(\mathrm{kg} \text { or } \mathrm{g}) \\
\mathrm{f} & =\text { tension }(\mathrm{kgf} \text { or } \mathrm{gf}) \\
\mathrm{a} & =\text { angle of forming } \alpha \\
\mathrm{b} & =\text { angle of forming } \beta
\end{array}
$$

The mechanical design of the sensor used consists of 2 main parts: 1) the plate is made oblique with a length of $8 \mathrm{~cm}$ in one part with a plastic tension and dancer roll and 2) inductive proximity sensor with analog output. The design of the speed sensor system is shown in figure 5.

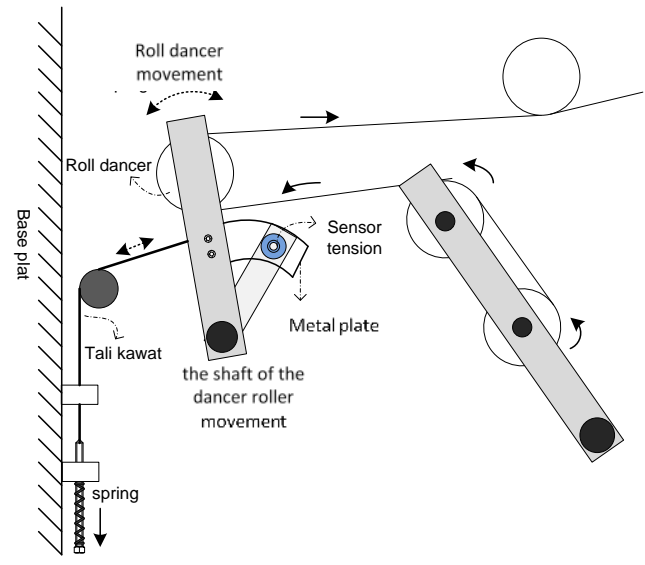

Fig. 5. The tension sensor system.

Figure 5 illustrates the design of the roll dancer which includes the position of the tension sensor, the metal plate of the roll position of the dancer and the addition of resistors to the tension sensor wiring. As the plastic sheet material undergoes a tension change, it will change the relative position of the metal plate against the proximity switch. The relative position between the metal plate and the proximity switch representing the tension of the plastic sheet material is detected by the proximity switch to produce an output voltage of 0-10 VDC. 


\subsection{PID controller}

The PID controller is a combination of three basic proportional control (P), integral control (I) and derivative control (D). The PID diagrams of the parallel structures used are shown in Figure 6 [7]. High reliability, economical cost, and ease of control have caused the application of dc motor in industry very popular. Setting the voltage on the armature or the field is two ways in controlling the speed of the motor dc rotation. One of the most widely used controllers is PID. A lot of development against PID controllers, such as combining it with fuzy logic (Reza Akbari-Hasanjani, Shahram Javadi, 2015).

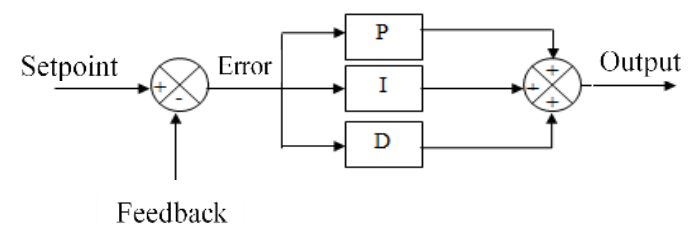

Fig. 6. Parallel PID Structure

The equation describing the PID controller action is [10] :

$$
\mathrm{u}(\mathrm{t})=\mathrm{K}_{\mathrm{P}}\left\{\mathrm{e}(\mathrm{t})+\frac{1}{\mathrm{~T}_{\mathrm{I}}} \int \mathrm{e}(\mathrm{t}) \mathrm{dt}+\mathrm{T}_{\mathrm{D}} \frac{\mathrm{de}(\mathrm{t})}{\mathrm{dt}}\right\}
$$

As description:

$$
\begin{aligned}
& \mathrm{u}(\mathrm{t})=\text { PID Control Output } \\
& \mathrm{e}(\mathrm{t})=\text { error } \\
& \mathrm{K}_{\mathrm{P}}=\text { proportionalgain } \\
& \mathrm{T}_{\mathrm{I}}=\text { Integral time } \\
& \mathrm{T}_{\mathrm{D}}=\text { derivative time }
\end{aligned}
$$

One use of PID controls is in the form of independent modules on the PLC system. Figure 7 is a function block diagram of PID on Siemens S7-1200 PLC.

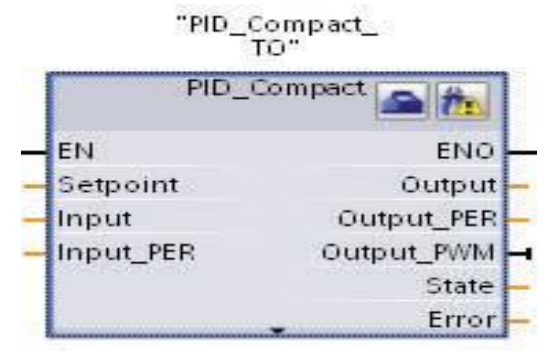

Fig. 7. PID on Siemens S7-1200 PLC [9]. 
The PID instruction on the PLC provides PID control with integrated tuning for both automatic and manual mode. The PID_Compact function block algorithm used in the calculation follows the following equation [9].

$$
y=K_{P}\left[b(w-x)+\frac{1}{T_{i} \cdot s}(w-x)+\frac{T_{D} \cdot s}{a \cdot T_{D} \cdot s+1}(c . w \cdot x)\right]
$$

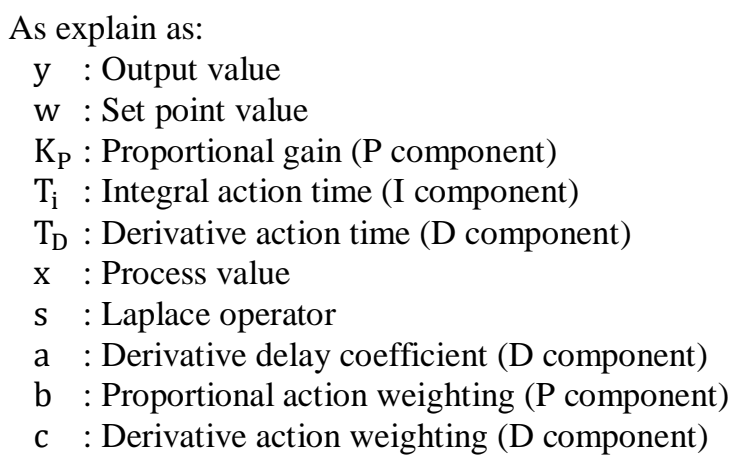

\section{Result and Analysis}

\subsection{Sensor Tension Testing}

The sensor used to detect tension is inductive proximity with 4-20 mA analog output. To convert the sensor output current (4-20 mA) into a voltage $(0-10 \mathrm{VDC})$ then a $500 \mathrm{ohm}$ resistor is installed in parallel at the sensor output. Tension sensor testing is done by placing the object (metal) in front of the proximity sensor. In this case it is done by using a bolt that is placed permanently or locked so that the male bolts move steadily when rotated. This bolt rotation determines the distance between the sensor and the end of the bolt. So that the inductance on the sensor can change as the bolt distance with the sensor, so it will affect the current and output voltage of the sensor. The voltage output voltage (0-10 VDC) of the next tension sensor will be fed as input for the PLC to be converted into an integer (16 bits). But integer data cannot be processed in PLC program, then converted again into real data (32 bits) by using function NORM_X and set its range 0 to minimum and 27648 for maximum. From NORM_X we change the scale to from -5 to 105 according to system data requirements. Tension sensor testing is shown in Fig. 8. 


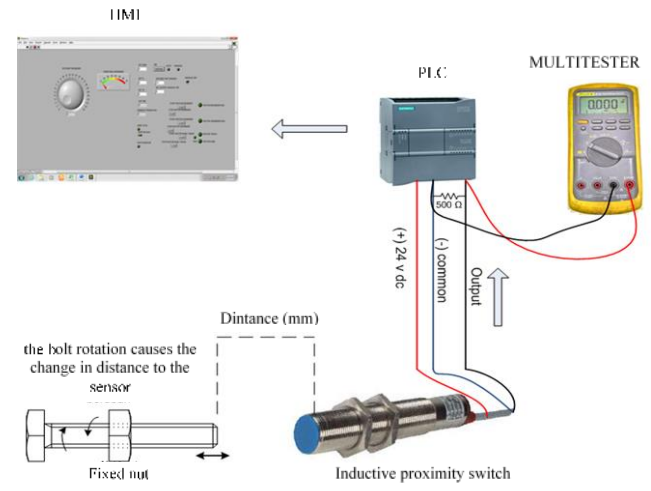

Fig. 8. Tension sensor testing.

Graph of system response as shown in figure 9 as follows:

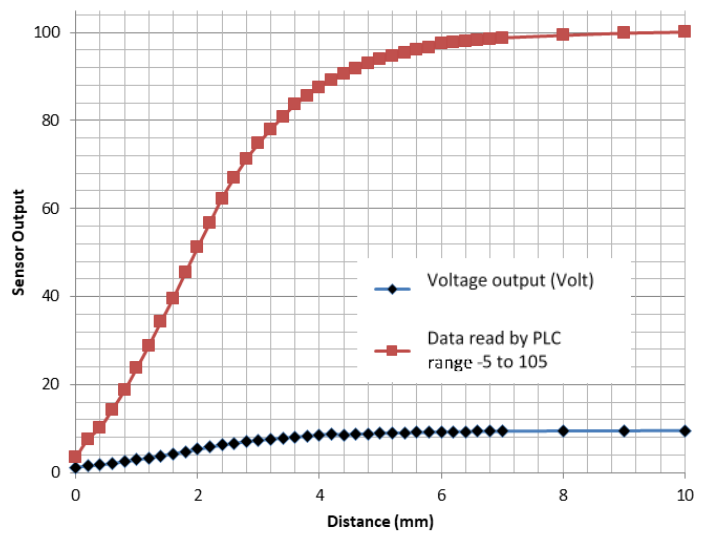

Fig. 9. Sensor response to distance change.

Figure 9 shows the nonlinearity of the sensor output response to the change in input distance. So it will have difficulty when entering the control on the PLC [11]. Then the sensor calibration required to find the results of a liner so that it will facilitate the control of system wrapping machine.

\subsection{Tension Sensor Calibration}

The tension sensor calibration is performed to obtain the tension value data with the actual strong pull strength using the weighed load. The purpose of this calibration is to set the pull of the dancer roll to the actual load according to the plastic strength range so that the data obtained on the plc matches the actual load and finds the linear data to facilitate the control of the system. How to perform a tension calibration on a rewinder system: 
1. Use a rope that is $50 \mathrm{~cm}$ long.

2. Tie the strap on the roll of plastic material, then insert the rope as per the plastic path on the unwinder system.

3. At the end of the rope is made a hook for the burden (iron).

4. Put the load gradually and record the result. The range used is the maximum plastic strength of $0-2.4 \mathrm{~kg}$.

5. For calibration, in the mechanical part there are bolts in the spring that can be adjusted the distance / density and change the position of the tension sensor to the dancer roll. Set so get range $0-2,4 \mathrm{~kg}$.

6. In the plc program part is also added to zero-position no load and increase the range so get range $0-100 \%$ in PLC data for load $0-2,4 \mathrm{~kg}$.

The calibration of the tension sensor is shown in Figures 10 and 11.

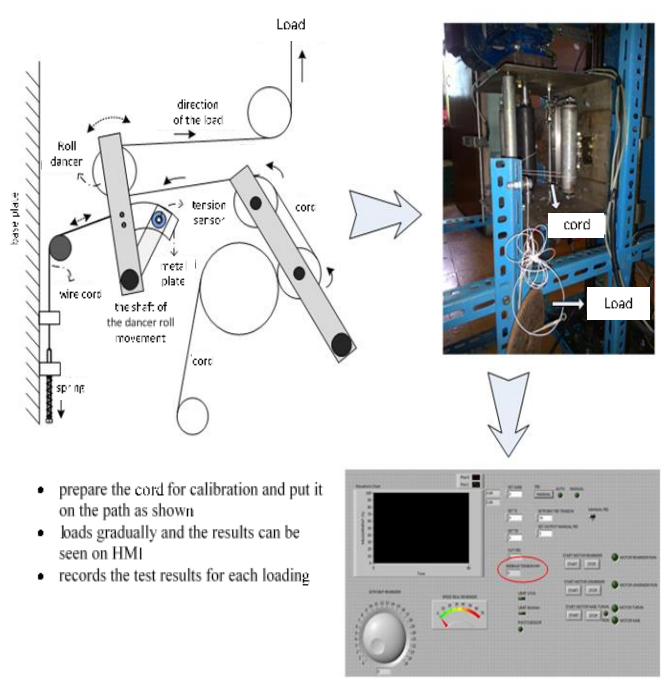

Fig. 10. Tension sensor calibration process.

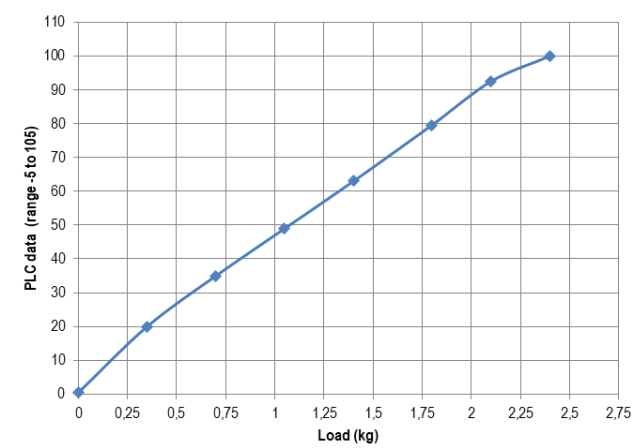

Fig. 11. Sensor response (after calibration). 
Graphic Figure 11 shows a linear result that will make it easier to control the wrapping machine system [11].

\subsection{PID Tuning With Reaction Curve Method}

PID tuning aims to determine the exact amount of $\mathrm{Kp}$, Ti and $\mathrm{Td}$ in order for the controller to produce the correct control signal in controlling the motor spin. The tuning by the curve reaction method is performed by giving a change in motor speed setpoint or in this case is done by changing the auto PID mode with manual PID. In the PID manual, Setpoint PID (0$100 \%)$ equals the PID output (0-100\%). Analog output PLC module output voltage 0-10 VDC as reference motor speed unwinder. From this setpoint change will result in the speed of the motor is changed so that the plastic tension also changed. Process data recorded by data logger to be analyzed.

Setpoint change $=90 \%$ to $50 \%$

Tension change that happened $=0 \%$ to $72 \%$

Response of setpoint input system and output tension can be seen in figure 12 .

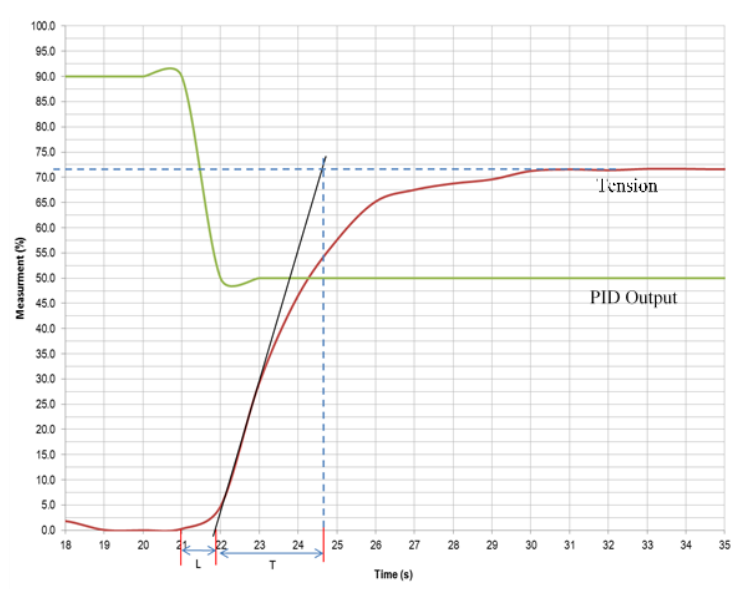

Fig. 12. Tension response to manual PID set point.

From the curve in Figure 12 we can know the parameters $\mathrm{L}$ and $\mathrm{T}$ are each of $0.9 \mathrm{~s}$ and $2.8 \mathrm{~s}$, so the magnitude of control parameters can be determined as follows :

$$
\begin{gathered}
K_{P}=1,2\left(\frac{\mathrm{T}}{\mathrm{L}}\right)=1,2\left(\frac{2.8}{0.9}\right)=3,73 ; \\
T_{i}=1,2 . \mathrm{L}=1,2(0,9)=1,08 \mathrm{~S} ; \\
T_{d}=0,5 . \mathrm{L}=0,5(0,9)=0,45 \mathrm{~S}
\end{gathered}
$$

The result of testing of system control with parameter of PID controller using tuning of curve reaction method is as in figure 13 . 


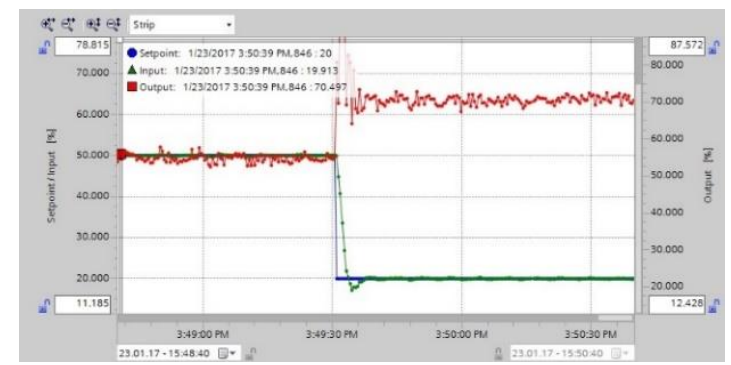

(a)

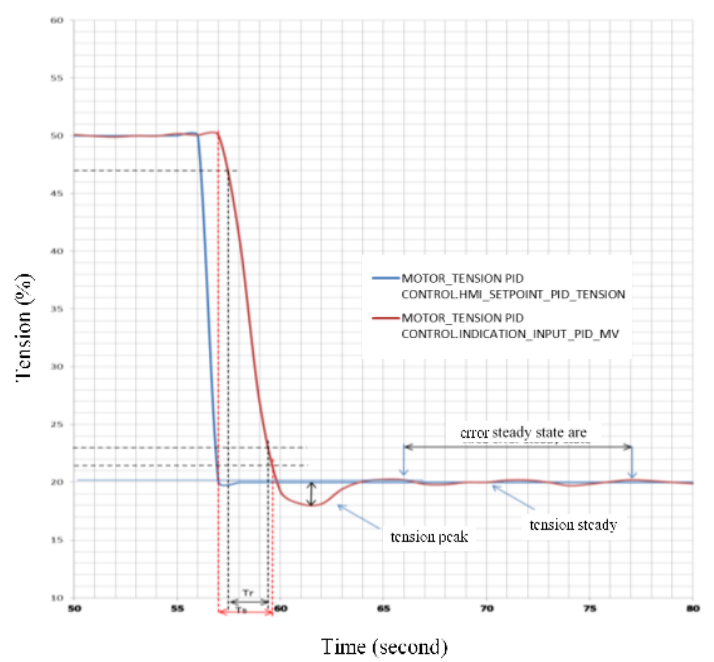

(b)

Fig. 13. system response to setpoint changes from $50 \%$ to $20 \%$.

From the graph of figure 13 it can be known the characteristics of the system output is as follows:

$$
\begin{gathered}
\% \text { Overshoot }=\frac{\mid \text { Tension }_{\text {puncak }}-\text { Tension }_{\text {steady }} \mid}{\text { Tension }_{\text {steady }}} \times 100 \% \\
=\frac{|18-20|}{20} \times 100 \% \\
=0.1 \times 100 \% \\
=10 \%
\end{gathered}
$$

The rise time $\left(t_{r}\right)$ is the time it takes for the output to change from $10 \%$ to $90 \%$ of steady state output : 
The setling time (ts) is the time it takes for the output to change from $0 \%$ to $95 \%$ of steady state output :

$$
\mathrm{t}_{\mathrm{s}} \quad=4 \mathrm{~s}
$$

The number of oscillation $=1$

Steady state error :

$$
\begin{gathered}
\% E_{S S} \quad=\frac{1}{n} \sum_{i=1}^{n}\left|\frac{\text { Output }(i)-\text { setpoint }}{\text { setpoint }}\right| \times 100 \% \\
=\frac{1}{36}|0.235| \times 100 \% \\
=0.00653 \times 100 \% \\
=0.653 \%
\end{gathered}
$$

From the analysis result shows that when the system is given set point change from $50 \%$ to $20 \%$, obtained the system output response with overshoot of $10 \%$, the rise time of 2 seconds, settling time of 4 seconds, 1 oscillation and steady state error $0.653 \%$. The system is able to control the output according to the given set point.

\section{Conclusion}

Based on the analysis of the test results presented earlier it can be concluded that the tension sensor used has nonlinear characteristics, so that calibration is done. From sensory calibration results have been obtained linear sensor characteristics. PID tuning with reaction curve method has resulted PID parameter equal to $\mathrm{Kp}=3.73 ; \mathrm{Ti}=1.08 \mathrm{~s}$; and $\mathrm{Td}=0.45 \mathrm{~s}$, and indicates its ability to control system output following the given set point. When the system is given set point changes from $50 \%$ to $20 \%$, the system output response with overshoot is $10 \%$, the rise time is 2 seconds, and the settling time is 4 seconds, 1 oscillation and steady state error $0.653 \%$.

Acknowledgements. We thank our colleagues of the Budi Luhur Research Team who has helped in completing the research and the preparation of the paper.

\section{References}

[1]M. S. Sani and F. A. Aziz, "Advanced manufacturing systems in food processing and packaging industry,” IOP Conf. Ser. Mater. Sci. Eng., vol. 46, no. 1, 2013.

[2]M. Boutaous, P. Bourgin, and D. Knittel, "Online Optimization of the Control Tension in Winding Systems," IFAC Proc. Vol., vol. 37, no. 15, pp. 311-316, 2004.

[3]J.-S. Lu, M.-Y. Cheng, M.-C. Tsai, and K.-H. Sua, "Wire tension control of an automatic motor winding machine - an iterative learning sliding mode control approach," Robot. Comput. Integr. Manuf., vol. 50, pp. 50-62, 2018.

[4]G. Cherubini, A. Pantazi, and M. Lantz, "Near-optimal tape transport control with feedback of velocity and tension," IFAC-PapersOnLine, vol. 49, no. 21, pp. 19-25, 2016.

[5]V. R. Khramshin, A. A. Radionov, G. P. Kornilov, and K. E. Odintsov, "Improvement of Electric and Mechanical System for Automated Strip Tension Control at Continuous Wide-Strip Hot-Rolling Mill," Procedia Eng., vol. 150, pp. 11-17, 2016.

[6]H. Yang, J. B. C. Engelen, A. Pantazi, W. Häberle, M. A. Lantz, and S. Müftü, "Mechanics of lateral 
positioning of a translating tape due to tilted rollers: Theory and experiments,” Int. J. Solids Struct., vol. 66, pp. 88-97, 2015.

[7]A. E. N. Gene F Franklin, J David Powell, Feedback Control of Dynamics Systems. USA: Edisson Wesley, 1987.

[8]Sujono and E. Yan, "Modelling and Controlling Level of Stress and Displacement Speed at Sheet Metal Processor," in the 12th International Conference on Quality in Research (QiR), 2011, no. Prosiding, p. 979.

[9]Siemens, "MODULE M6 Control Engineering using the SIMATIC S7-1200 with TIA PORTAL V $10, "$ Germany, 2011.

[10]R. S.-N. Reza Akbari-Hasanjani, Shahram Javadi, "DC motor speed control by self-tuning fuzzy PID algorithm," SAGE J., vol. 37, no. 2, pp. 164-176, 2015.

[11]Siemens, "MODULE M1 'Startup' Programming of the SIMATIC S7-1200 withTIA Portal V10," Germany, 2011. 